\title{
Contribution of Primary Spikes Vs Tillers to Total Deoxynivalenol in Harvested Grain of Wheat and Barley
}

\author{
${ }^{1}$ Pravin Gautam, ${ }^{2}$ Scott Halley and ${ }^{1,3}$ Jeffrey M. Stein \\ ${ }^{1}$ Department of Plant Science, South Dakota State University, Brookings, SD, USA \\ ${ }^{2}$ Langdon Extension Research Center, North Dakota State University, Langdon, ND, USA \\ ${ }^{3}$ Monsanto Company, Waterman, IL, USA
}

Received 2012-05-10, Revised 2012-05-27; Accepted 2012-07-23

\begin{abstract}
Formation of higher number of tillers is desirable for higher grain yield in both wheat and barley; tillers have delayed physiological development. Studies addressing the contribution of secondary tillers in final DON concentration for wheat and barley are limited. This project aims to improve our understanding of the relationship between FHB symptoms and DON concentration by examining the contribution of primary spikes and secondary tillers as it relates to the final DON concentration. Experiments, established as split-split plot design with five replications, was be conducted in South Dakota with moderately resistant and susceptible cultivars of each spring wheat, winter wheat and barley. Two inoculation time treatments was applied; at the anthesis of primary spikes (Feekes 10.5) and at anthesis of tillers (Feekes 11.2). Primary spikes (inoculated at Feekes 10.5) and tillers (inoculated at Feekes 11.2) were harvested separately, analyzed for visually scabby kernels and deoxynivalenol following standard protocols. Levels of DON were statistically higher in main heads of barley and spring wheat in each cultivar compared to its tillers. In winter wheat, though there was higher DON level in tillers than main heads in each cultivar, it was not statistically different. Based upon our result, it is suggested that cultivars with high tillers number should be selected in breeding programs to develop cultivars with high yield and low DON. Similarly, planting density of wheat and barley can be adjusted such that it will result in higher numbers of tillers, increasing tillers proportion that might lower DON concentration in final harvest.
\end{abstract}

Keywords:Fusarium Head Blight (FHB), Vomitoxin, Late Infection, Gibberella Zeae, Fusarium Graminearum, Fhbl

\section{INTRODUCTION}

Development of higher numbers of tiller has been linked to positive impact on final grain yield of both wheat and barley. Nerson (1980) reported a more than double increase in wheat yield when tiller numbers were increased from 6 to 16 per plant despite the decrease in plant density by about 70\%. Dofing and Knight (1992) conducted a path analysis for barley grain yield and reported a negative impact of increased number of spikes per square meter on two yield components: Kernel weight and kernel per spike. However, the impact on yield was highly positive, resulting in a net effect of increased yield.

Fusarium Head Blight (FHB) of wheat and barley in North America is primarily caused by Gibberella zeae
(Schwein) Petch [anamorph F. graminearum Schwabe] (Bai et al., 2001). The protrudence of anthers (Strange and Smith, 1971) and temporary opening of florets during anthesis are strongly linked to susceptibility of wheat to infection by Fusarium spp. (Bushnell et al., 2003).

Infection occurring at anthesis results in higher disease symptoms and DON concentration in the final grain, however, infection by Fusarium spp. can occur even up to the hard dough stage of wheat (Ponte et al., 2007). Late infections typically result in lower levels of symptomatic grain than infection which occur during anthesis. Interestingly, Cowger and Arrellano (2010) reported high DON concentration in late infected winter wheat in the presence of high moisture conditions, despite the presence of a low level of visually scabby kernels. The 
situation of low disease-high DON is pronounced more under the situation of delayed or prolonged crop development. Reasons behind the high DON level in grain of crops which have undergone delayed crop development and/or late infection are not fully understood. We assume that due to later maturation of spikes on tillers than main stem, tillers might create the situation analog to the late infection with impact on the observed low FHB-high DON condition. The growth and development of spikes on tillers is slightly delayed as compared to the main stem (Klepper et al., 1998). Differential growth and maturation of spikes within the same plant or even same field extends the window of infection by $G$. zeae. However, studies addressing the contribution of secondary tillers in final DON concentration are limited.

Past studies have indicated the peaking of DON in grain sometime before maturation followed by a decline or steady levels thereafter. Scott et al. (1984) reported a decline in DON levels before harvest in naturally infected commercially grown winter wheat in Ontario, Canada. They observed a decline in the DON levels as the grain matured and the decrease was consistent across all fields evaluated. Miller and Young (1985) also reported that DON peaked six weeks after infection and declined thereafter until it reached a constant level before harvest. Others have also reported DON peaking in wheat and declining thereafter (Argyris et al., 2003; Culler et al., 2007). A similar decline in DON levels before harvest was reported in barley. However, high DON levels in final harvest despite low disease symptoms might be due to the delay in physiological development of secondary tillers compared to the primary spikes, in turn delaying the spiking and decline of DON levels. Similarly, since malt barley is not free threshing, late infections of the palea and lemma could impact DON at harvest.

An understanding of the contribution of different tillers to the final DON level in Fusarium-infected wheat and barley heads can be used in disease management. For example, farmers can manage FHB and DON by choosing cultivars with low or high tiller number. Furthermore, tiller numbers can be manipulated by adjusting planting density and knowledge obtained from this study might also be useful to breeders so that they can consider tillering characteristics in FHB resistant cultivar breeding. The results from this experiment might also be useful to chemical companies for fungicide development by encouraging shorter pre-harvest intervals. Finally, the result might also help in decision-making process of farmers to identify the most ideal time for fungicide application and to assess the economics of sequential fungicide applications.

The objective of this study was to improve our understanding of the relationship between Fusarium Head Blight (FHB) symptoms and DON concentration by examining the contribution of primary spike and secondary tillers as it relates to the final DON concentration.

\section{MATERIALS AND METHODS}

Experiments were conducted at the South Dakota State University's Plant Pathology Farm in Brookings, $\mathrm{SD}$ in 2011. The experimental design was a split-split plot design with five replications. Two inoculation treatments (inoculation at anthesis of primary spikes or at anthesis of lateral tillers) were applied as main plots; cultivars were on the sub-plots and the sub-sub plot was G. zeae or mock inoculations. Assignments of treatments were random. Each experimental unit was a plot of $1.5 \mathrm{~m}$ $\mathrm{x} 4.5 \mathrm{~m}$. The plots were planted in mid-May. Bronate ${ }^{\circledR}$ (3, 5-dibromo-4-hydroxybenzonitrile, 0.086 L a.i./ha; Bayer Crop Science, Research Triangle Park, NC) was applied at the 3-4 leaf stage to control broadleaf weeds and Di-syston $8 \AA$ (disulfoton, 0.082 L a.i./ha; Bayer Crop Science, Research Triangle Park, NC), was applied to control aphids and other insects. Hand weeding supplemented chemical weed control.

\subsection{Plant Materials}

The experiment included three different crops; barley, Hard Red Spring Wheat (HRSW) and Hard Red Winter Wheat (HRWW). Two each of barley; 'Robust' [Susceptible (S) to FHB] and 'Quest' [moderately resistant (MR)]; hard red spring wheat; 'Brick' (MR) and SD3854 (MS) and hard red winter wheat; WesleyBC6 (MR) and WesleyBC70 (MS) were used in the study. Within each wheat class, the two entries were closely related genetically and differed by the fact that the MR entry possessed the Fhbl QTL for Type II resistance and the $S$ entry did not. In all cases the pairs were similar agronomically, e.g., flowering date, height.

\subsection{Inoculum Production}

Ten single-spored isolates of $G$. zeae, were used and inoculum was produced following the protocol of Gautam and Dill-Macky (2012). Briefly, isolates were cultured on mung bean agar media (MBA) in petri dishes (40 g mung beans boiled for $23 \mathrm{~min}$ in $1000 \mathrm{~mL}^{-1}$ of Deionized (DI) water, filtered through two layers of gauze pads, adjusted to $1 \mathrm{~L}$ with DI water, $15 \mathrm{~g}$ of Difco agar [Bectin, Dickinson and Company, Sparks, MD 21152] and autoclaved). Isolates in MBA were allowed to grow for 7 days under fluorescent and UV lights (12 h: $12 \mathrm{~h}$ light: Dark cycle) at room temperature $\left(22-24^{\circ} \mathrm{C}\right)$. On the seventh day, macroconidia were harvested by washing MBA plates with ca. $20 \mathrm{~mL}^{-1}$ of deionized (DI) water per plate using a $\mathrm{CO}_{2}$ powered backpack sprayer fitted with flat-fan spray tip (TeeJet SS8003; Spraying Systems Co., Wheaton, IL) at an operating pressure of ca. $276 \mathrm{kpa}$. The spore suspension was filtered through one layer of cheesecloth to remove mycelia fragments. The spore concentration was determined using a hemacytometer. The inoculum concentration was adjusted to $18 \times 10^{4}$ spores $\mathrm{mL}^{-1}$ and stored at $-80^{\circ} \mathrm{C}$ until 
used for inoculation. The inoculum was diluted to $9 \times 10^{4}$ spores $\mathrm{mL}^{-1}$ for inoculation before use.

\subsection{Inoculation}

Inoculums viability was tested by plating $0.5 \mathrm{~mL}$ of stock inoculum on Potato Dextrose Agar (PDA) and counting germinated spore after $8 \mathrm{~h}$. We intended not to use inoculum with less than $90 \%$ spore germination and this will reduce the variability arising due to the difference in spore germination. However, in our case all inoculum had more than $95 \%$ spore germination.

Twenty-five primary spikes or tillers were tagged as appropriate when the plots were at primary spike (Feekes $10.5)$ and tiller ( Feekes 11.2) anthesis, respectively for wheat and at full spike emergence for $50 \%$ of the primary spikes and tillers, respectively for barley. These were inoculated with a suspension of macroconidia (ca. $9 \times 10^{4}$ spores $\left.\mathrm{mL}^{-1}\right)$ and polysorbate $\left(2.5 \mathrm{~mL}^{-1}\right)$. Inoculum was applied using a $\mathrm{CO}_{2}$-powered backpack sprayer, operating at a pressure of ca. $276 \mathrm{kPa}$ and fitted with flat-fan spray tip, at a rate of $29.6 \mathrm{~mL}$ per square meter area. Control plots were mock inoculated with water. A mist-irrigation system was run for $10 \mathrm{~min}$ immediately following inoculation in order to facilitate infection. This system, mounted on risers at a height of $1 \mathrm{~m}$, was programmed to run for 10 min every hour starting at 5 p.m. and ending at 7 a.m. Misting was applied until 14 days after inoculation (dai).

\subsection{Sampling, Disease Assessment and Mycotoxin Analysis}

FHB severity was assessed visually 18 dai by counting total and the number of infected spikelets on tagged main spikes and tillers. At maturity, tagged spikes from individual plots were manually harvested, threshed, bulked and analyzed for Visually Scabby Kernels (VSK) by enumerating the number scabby kernels in 100 seeds (3 reps). The rest of the plots were mechanically harvested at maturity and threshed using a low fan speed in order to retain heavily infected grain kernels and dried for 4 days at $95^{\circ} \mathrm{C}$. Dried grain was cleaned using a belt thresher and test weight and yield were estimated. In barley, since VSK could not be assessed, seeds were passed through seed separator delivering wind at $30 \mathrm{mph}$ and separated into four categories ( 1 to 4 ; lightest to heaviest) based upon seed weight. H/L Ratio was calculated using formula; H/L Ratio $=(3+4) /(1+2)$. Following the assessment of VSK, the samples were ground and submitted for mycotoxin analyses to Veterinary Diagnostic Laboratory, North Dakota State University. DON and related mycotoxins was analyzed using gas chromatography-electron capture detector (GC$\mathrm{ECD}$, detection limit $0.5 \mathrm{ppm}$ ). Test weight was measured using a standard USDA 1 pint cup and converted to $\mathrm{kg} \mathrm{hL}^{-}$ ${ }^{1}$. Yield $\left(\mathrm{kg} \mathrm{ha}^{-1}\right)$ was estimated from the total yield obtained from individual plot.

\subsection{Data Analyses}

Arcsine transformed data of barley severity and HRWW FDK, log transformed barley DON data and square root transformed HRSW FDK and DON data were used for analyses. Data on HRSW severity, HRWW severity and DON; and test weight and yield of all three crops were analyzed without transformation. Data were analyzed using proc mixed procedure in SAS v. 9.2 (SAS Institute Inc., Cary, NC). Means were separated using Fisher's LSD.

\section{RESULTS}

\subsection{Disease Severity}

FHB severity in mock inoculated plot was relatively high (up to $50 \%$ ) for the study. It was significantly lower than the Geae inoculated plots.

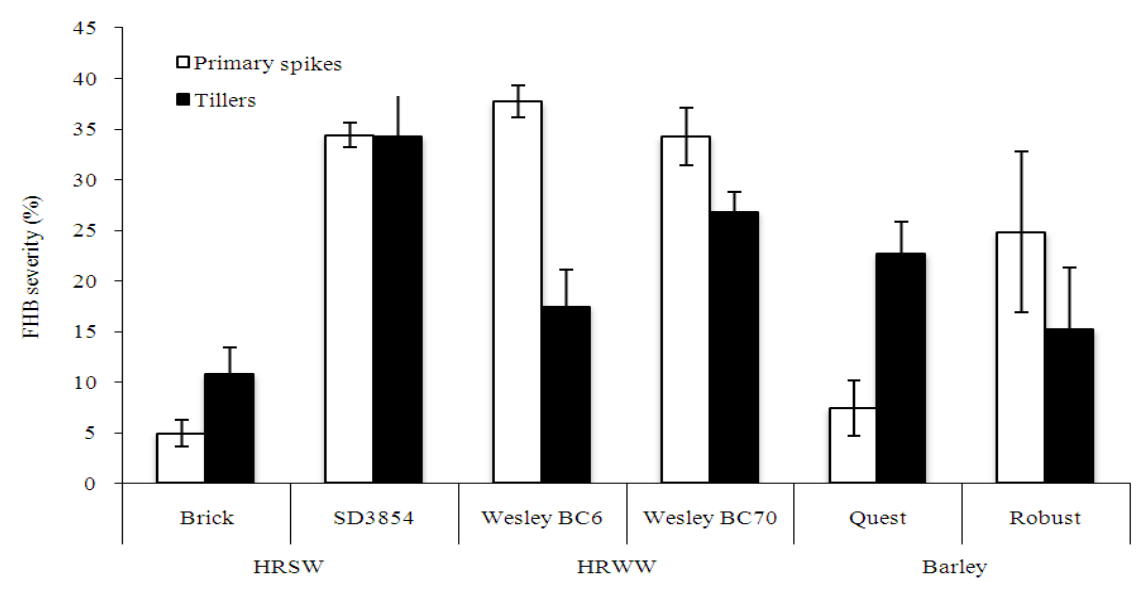

Fig. 1. FHB severity (\%) observed in primary spikes and tillers of HRSW [Brick (MR) and SD3854 (MS)], HRWW [Wesley BC6 (MR) and Wesley BC70 (MS)] and barley [Quest (MR) and Robust (MS)] 
Pravin Gautam et al. / American Journal of Agricultural and Biological Sciences 7 (3) (2012) 293-300

Table 1. Analysis of variance of Fusarium Head Blight (FHB) severity, H/L Ratio, Visually Scabby Kernels (VSK) and Deoxynivalenol (DON), of head samples harvested at maturity of barley, Hard Red Spring Wheat (HRSW) and Hard Red Winter Wheat (HRWW)

\begin{tabular}{|c|c|c|c|c|c|c|c|c|c|c|c|c|c|c|c|}
\hline \multirow[b]{2}{*}{ Effects } & \multicolumn{5}{|l|}{ Barley } & \multicolumn{5}{|c|}{ Hard Red Spring Wheat (HRSW) } & \multicolumn{5}{|c|}{ Hard Red Winter Wheat (HRWW) } \\
\hline & Severity & H/L Ratio & DON & Test wt. & Yield & Severity & VSK & DON & Test wt. & Yield & Severity & VSK & DON & Test wt. & Yield \\
\hline Spike types (ST) & $0.32^{\mathrm{NS}}$ & $0.99^{\mathrm{NS}}$ & $170.95^{* *}$ & $1.66^{\mathrm{NS}}$ & $1.00^{\mathrm{NS}}$ & $1.12^{\mathrm{NS}}$ & $5.64 *$ & $34.92^{* *}$ & $12.17^{* *}$ & $16.40^{* *}$ & $28.23 * *$ & $4.69^{\mathrm{NS}}$ & $0.63^{\mathrm{NS}}$ & $12.76^{* *}$ & $0.59^{\mathrm{NS}}$ \\
\hline Cultivar (Cult.) & $0.42^{\mathrm{NS}}$ & $27.23 * *$ & $18.11^{* *}$ & $2.60^{\mathrm{NS}}$ & $2.53^{\mathrm{NS}}$ & $97.92 * *$ & $0.71^{\mathrm{NS}}$ & $0.01^{\mathrm{NS}}$ & $22.27 * *$ & $67.26^{* *}$ & $1.29^{\mathrm{NS}}$ & $1.09^{\mathrm{NS}}$ & $0.37^{\mathrm{NS}}$ & $0.04^{\mathrm{NS}}$ & $2.20^{\mathrm{NS}}$ \\
\hline ST*Cult. & $5.99 *$ & $1.70^{\mathrm{NS}}$ & $10.87 * *$ & $0.12^{\mathrm{NS}}$ & $1.76^{\mathrm{NS}}$ & $1.23^{\mathrm{NS}}$ & $15.55^{* *}$ & $6.29 *$ & $7.63^{*}$ & $1.48^{\mathrm{NS}}$ & $6.10^{*}$ & $1.13^{\mathrm{NS}}$ & $0.09^{\mathrm{NS}}$ & $2.23^{\mathrm{NS}}$ & $0.01^{\mathrm{NS}}$ \\
\hline
\end{tabular}

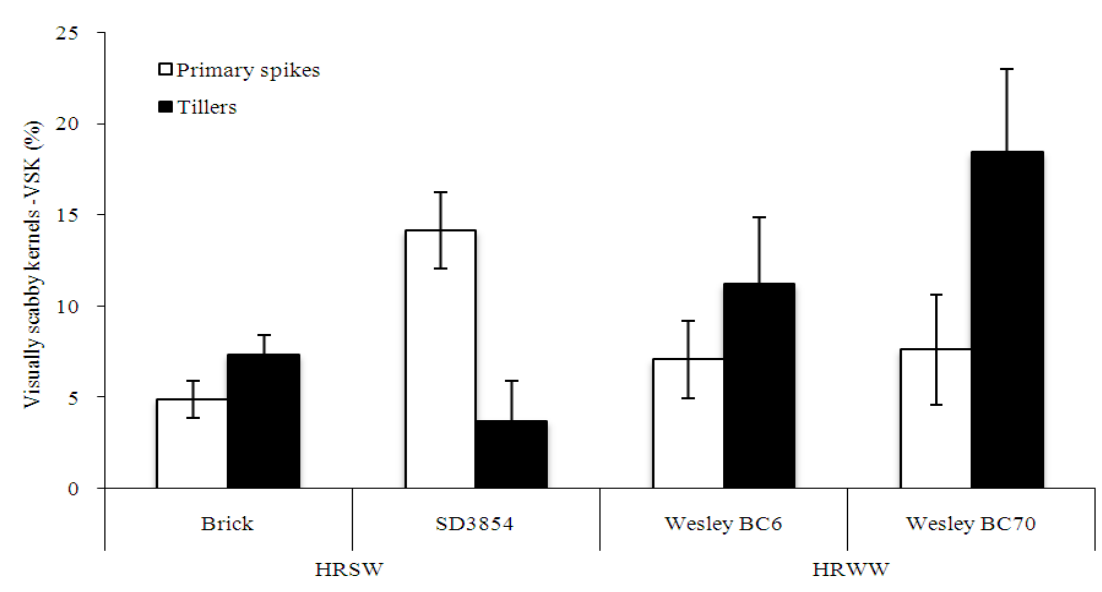

Fig. 2. Visually scabby kernels (VSK, \%) observed in primary spikes and tillers of HRSW [Brick (MR) and SD3854 (MS)] and HRWW [Wesley BC6 (MR) and Wesley BC70 (MS).]

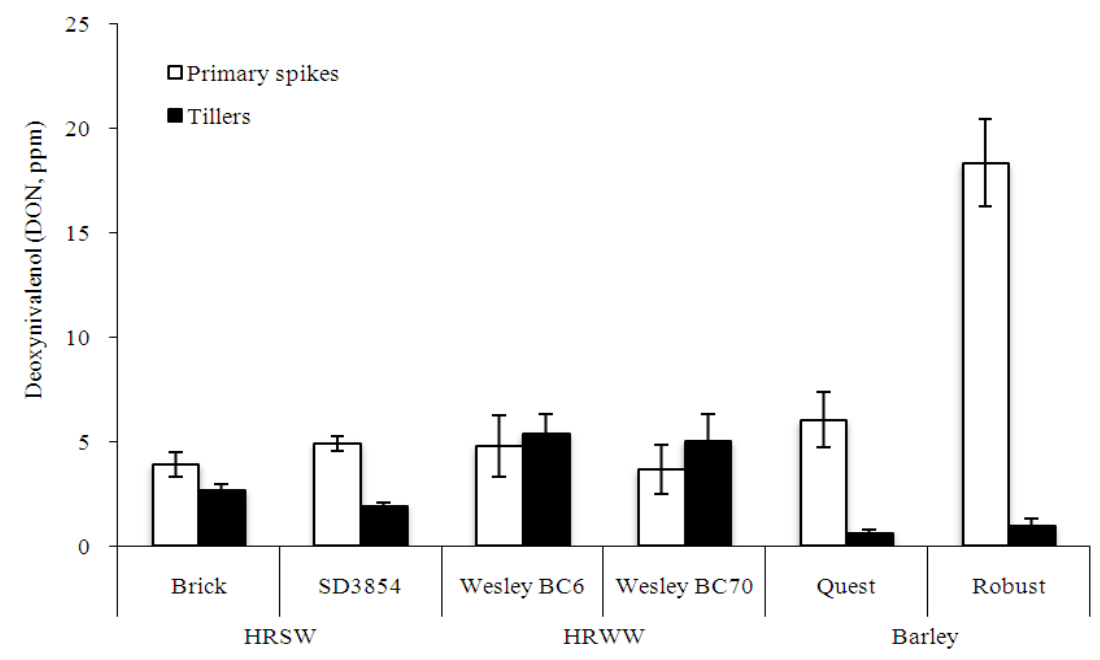

Fig. 3. Deoxynivalenol (DON, ppm) observed in primary spikes and tillers of HRSW [Brick (MR) and SD3854 (MS)], HRWW [Wesley BC6 (MR) and Wesley BC70 (MS)] and barley [Quest (MR) and Robust (S)].

In barley, FHB severity was not statistically different between primary spikes and tillers or between cultivars (Table 1); however, the interaction of cultivars with spike types was significant. Robust had significantly higher FHB severity than Quest in primary spikes (Fig. 1). In HRSW, there was no statistical difference between FHB severity in primary spikes and tillers (Table 1). However, cultivar effect was significant with SD3854 (34.3\%) having significantly higher FHB severity than Brick (7.9\%). In HRWW, the main effect of spike types was significant, however, it was dependent on cultivar (Table 1). WesleyBC6 had significantly higher FHB severity in primary spikes than tillers, there was no difference within Wesley BC70 (Fig. 1). 


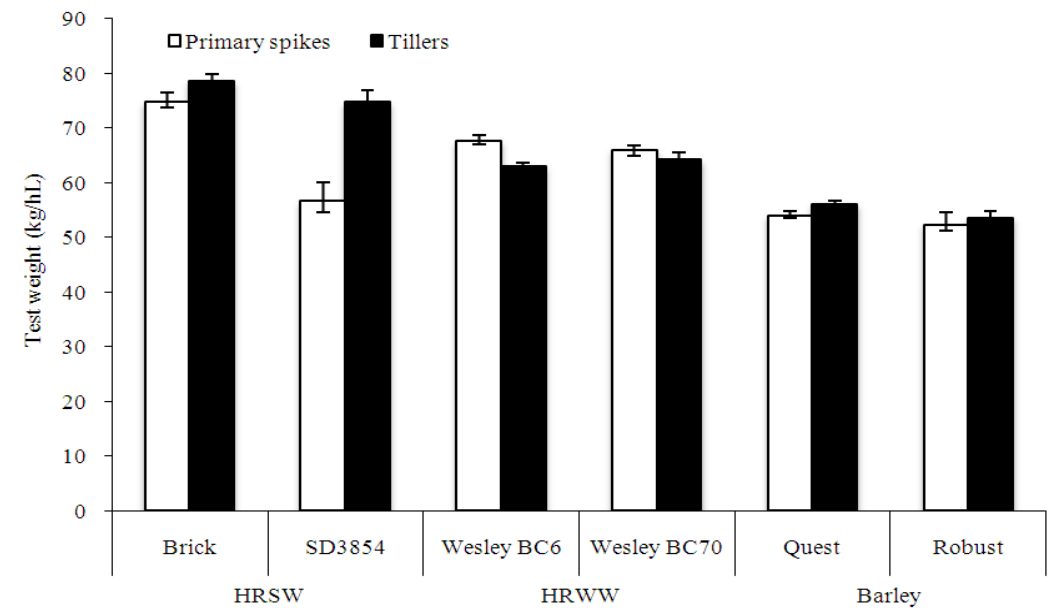

Fig. 4. Test weight (kg/hL) observed in primary spikes and tillers of HRSW [Brick (MR) and SD3854 (MS)], HRWW [Wesley BC6 (MR) and Wesley BC70 (MS)] and barley [Quest (MR) and Robust (S)].

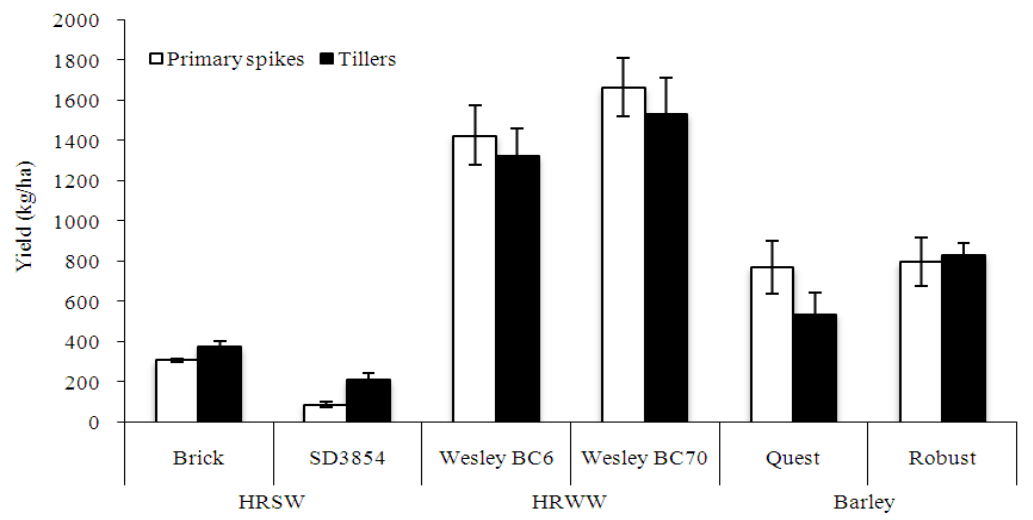

Fig. 5. Yield (kg/ha) observed in primary spikes and tillers of HRSW [Brick (MR) and SD3854 (MS)], HRWW [Wesley BC6 (MR) and Wesley BC70 (MS)] and barley [Quest (MR) and Robust (S)]

\subsection{H/L Ratio and Visually Scabby Kernel (VSK)}

Only the cultivar main effect was significant for $\mathrm{H} / \mathrm{L}$ ratio (Table 1) in barley. H/L ratio was significantly higher in Quest (0.86) compared to that of Robust (0.47). The effect of spike types was significant for VSK in HRSW; however, it was dependent on cultivars (Table 1). VSK was significantly higher in primary spikes of SD3854 compared to its tillers and the primary spikes and tillers of Brick (Fig. 2). In Brick there was no statistical difference between VSK observed in primary spikes and tillers. In HRWW, none of the variables were significant for VSK. Mean VSK in HRWW was $11.07 \%$.

\subsection{Deoxynivalenol}

All variables and their interactions were significant for DON in barley (Table 1). Levels of DON were statistically higher in primary spikes of barley in each cultivar compared to its tillers (Fig. 3). Robust had significantly higher DON levels than Quest only in primary spikes. In HRSW, DON levels were significantly higher in primary spikes than tillers in each cultivar; however, cultivars were not statistically different for DON levels. In HRWW, though there was higher DON level in tillers than primary spikes in each cultivar, it was not statistically different.

\subsection{Test Weight}

None of the variables had significant effect on test weight in barley (Table 1). Mean test weight in barley, combined over cultivars and inoculation, was $54.04 \mathrm{~kg}$ $\mathrm{hL}^{-1}$. All of the variables and their interactions were significant for test weight in HRSW. Test weight was significantly lower in primary spikes of SD3854 
compared to that of its tillers and primary spikes and tillers of Brick (Fig. 4). Brick had significantly higher test weight only in primary spikes compared to that of SD3854. In HRWW, only the spike types effect was significant. Test weight was significantly higher in primary spikes $(66.86 \mathrm{~kg}$ $\left.\mathrm{hL}^{-1}\right)$ than tillers $\left(63.6 \mathrm{~kg} \mathrm{hL}^{-1}\right)$.

\subsection{Yield}

None of the variables had significant effect on barley and HRWW yield (Table 1). Mean yield were 735 and $1488.1 \mathrm{~kg} \mathrm{ha}^{-1}$ for barley and HRWW, respectively (Fig. 5). For HRSW, only the main effects of spike types and cultivars were significant. Mean yield was significantly higher in tillers $\left(294.2 \mathrm{~kg} \mathrm{ha}^{-1}\right)$ than main heads (199.2 $\left.\mathrm{kg} \mathrm{ha}^{-1}\right)$ and significantly higher in Brick (342.9 kg ha $\left.{ }^{-1}\right)$ than SD3854 (150.5 $\left.\mathrm{kg} \mathrm{ha}^{-1}\right)$.

\section{DISCUSSION}

To the best of our knowledge, this is a first study to evaluate the contribution of primary spikes and secondary tillers to DON levels in harvest grains of three different crops with varying degree of resistance to FHB. Since no specific studies have been done in the past looking at the effect of tillers per se on FHB or any other disease, the result from this study cannot be compared directly with others. However, several studies have been conducted looking at wheat planting density and disease. In a study by Finckh et al. (1999), a negative correlation was found with powdery mildew and planting density in barley. Pfleeger and Mundt (1998) found little evidence for an effect of density on wheat leaf rust in wheat and wild oats mixtures and suggested this may have been because of compensatory tillering at low densities. In a two-year study, Garrett and Mundt (2000) reported an increase in severity of stripe rust caused by Puccinia striiformis in one year and decrease in next year with an increase in planting density.

It is a general assumption that higher number of tillers creates condition similar to high planting density in that latter promotes disease, especially foliar pathogens, due to a changed canopy microclimate. Ansar et al. (2010) reported an increase in Septoria leaf blotch severity in wheat with an increase in tiller number from 30 tillers per $\mathrm{m}^{2}$ to 600 tillers per $\mathrm{m}^{2}$. They attributed the increase in disease development at higher density due to a more favorable microclimate produced within the leaf canopy. Since, G.zeae is soil borne pathogen and its inoculum reach primarily due to rain splash from the ground level, it was our assumption that tillers might act as a barrier. Fewer spores would potentially reach primary spikes leading to higher infection and DON accumulation in tillers. Similarly, due to delayed physiological maturity of tillers; it was our assumption that tillers get infected with late arriving air borne inoculum and splashed inoculum. This might result in infection and higher DON levels compared to that of primary spikes. However, our result suggests that, despite delayed physiological development of tillers than primary spikes, primary spikes tend to accumulate higher proportion of DON. The reason behind high DON in primary spikes might be due to the fact that primary spikes reach anthesis earlier than tiller, thus fungus get longer period to grow and invade wheat tissue, leading to higher DON concentration.

Contribution of different spike types for yield was evaluated by Mass et al. (1996) and reported that under non-saline condition, main spikes contributed $25-35 \%$ and tillers contributed $62-83 \%$ of the total grain yield in wheat. Similarly several other reports (Nerson, 1980; Dofing and Knight, 1992) have indicated positive impact of tillers in total yield. Ansar et al. (2010) also reported an increase in total yield despite a decrease in individual grain weight and number of grains per spike when tillers were increased from 30 per $\mathrm{m}^{2}$ to 600 per $\mathrm{m}^{2}$. These increases in yield might be due to the additional grain obtained from tillers. Since they did not compare yield from primary spikes verses tiller, our results cannot be compared directly with theirs. In our study, no significant difference was observed in yield obtained from primary spikes with tillers except in HRSW, where estimated yield was significantly high in tillers. Though grains in primary spikes are generally larger and heavier than in tillers, in our case it was not evident. During the 2011 growing season and especially at the period of flowering and grainfill, the temperatures were unseasonably warm in the region, resulting into stunted plants and very small heads with abrupt anthesis. This might have resulted in smaller spikes and grains with lower test weight. It may also have influenced the resistance response of the host.

Several studies in the past reported that Fhbl QTL can significantly reduce FHB development. Pumphrey et al. (2007) reported an average reduction of $23 \%$ in disease severity and $27 \%$ for grain infection in NearIsogenic Lines (NILs) of wheat with the Fhbl resistant allele. Similarly reductions of kernel infection between 37 and $41 \%$ due to the effect of $F h b 1$ have been reported in different populations (Blaut et al., 2010). However, there were no significant differences in FHB severity due to Fhbl in HRSW and HRWW, except in the tillers of HRWW ( $\sim 35 \%$ reduction). Similarly, kernel infection or VSK was not statistically different in HRWW with and without Fhbl. However in HRSW, there was 32\% reduction in kernel infection or VSK when combined over primary spikes and tillers due to Fhbl. Inconsistent result obtained in our study are most likely due to the erratic environmental condition during the growing period. For example, the barley and HRSW planted past 
the optimal timeframe due to high soil moisture conditions on the farm, there were several heat waves at around anthesis stage, which is the key stage for infection by $G$. zeae. The unfavorable environmental condition also impacted yield and test weight. Yield of Brick in non-inoculated plot (444.28 $\mathrm{kg} \mathrm{ha}^{-1}$ ) was almost $86.8 \%$ less than the yield reported in cultivar registration by Glover et al. (2010).

\section{CONCLUSION}

Our results suggests that FHB severity tends to be higher in primary spikes than tiller in wheat when infection occurs under environment conditions condusive for infection. Similarly, primary spikes have larger contribution towards total DON levels at harvest than tillers. In periods of sub-optimal conditions, such as what was experienced by the HRSW and barley, it is likely that the contributions of the different spikes become more complicated. There was no difference in estimated yield between primary spikes and tiller (HRWW, barley) or high yield in tillers than primary spikes (HRSW). Thus based upon our results, it is suggested that cultivars with high tillers number should be selected in breeding programs to develop cultivars with high yield and low DON. Similarly, planting density of wheat and barley can be adjusted such that it will result in higher numbers of tillers, increasing tillers proportion that might lower DON concentration in final harvest.

\section{ACKNOWLEDGEMENT}

We would like to thank Mr. Richard Geppert for technical assistance and NDSU Veterinary Diagnostic Laboratory for DON testing. This material is based upon work supported by the U.S. Department of Agriculture, under Agreement No. 59-0206-9-060 and the South Dakota Wheat Commission. This is a cooperative project with the U.S. Wheat and Barley Scab Initiative. Any opinions, findings, conclusions, or recommendations expressed in this publication are those of the author(s) and do not necessarily reflect the view of the U.S. Department of Agriculture.

\section{REFERENCES}

Ansar, M., N.M. Cheema and M.H. Leitch, 2010. Effect of agronomic practices on the development of Septoria leaf blotch and its subsequent effect on growth and yield components of wheat. Paks. J. Bot., 43: 2125-2138.
Argyris, J., D.V. Sanford and D. TeKrony, 2003. Fusarium graminearum Infection during Wheat Seed Development and Its Effect on Seed Quality. Crop Sci., 43: 1782-1788. DOI: 10.2135/cropsci2003.1782

Bai, G.H., R. Plattner, A. Desjardin, F. Kolb and R.A. McIntosh. 2001. Resistance to fusarium head blight and deoxynivalenol accumulation in wheat. Plant Breed., 120: 1-6. DOI: 10.1046/j.14390523.2001.00562.x

Blaut, A., A. Clark, G. Brown-Guedira, E. Souza and D. Van Sanford, 2010. Validation of Fhbl and QFhs.nau-2DL in several SRW wheat breeding populations. Proceedings of the National Fusarium Head Blight Forum, Dec 7-9, University of Kentucky, Milwaukee, WI. Lexington, KY.

Bushnell, W.R., B.E. Hazen and C. Pritsch, 2003. Histology and Physiology of Fusarium Head Blight. In: Fusarium Head Blight of Wheat and Barley, Leonard, K.J. and W.R. Bushnell, (Eds.). APS Press, ISBN-10 : 089054302X, pp: 44-83.

Cowger, C. and C. Arrellano, 2010. Plump kernels with high deoxynivalenol linked to late Gibberella zeae infection and marginal disease conditions in winter wheat. Phytopathology, 100: 719-728. PMID: 20528190

Culler, M.D., J.E. Miller-Garvin and R. Dill-Macky. 2007. Effect of extended irrigation and host resistance on deoxynivalenol accumulation in fusarium-infected wheat. Plant Dis., 91: 1464-1472. DOI: 10.1094/PDIS-91-11-1464

Ponte, E.M.D., J.M.C. Fernandes and G.C. Bergstrom, 2007. Influence of growth stage on fusarium head blight and deoxynivalenol production in wheat. J. Phytopathol., 155: 577-581. DOI: 10.1111/j.14390434.2007.01281.x

Dofing, S.M. and C.W. Knight, 1992. Alternative model for path analysis of small-grain yield. Crop Sci., 32: 487-489.

Finckh, M.R., E.S. Gacek, H.J. Czembor and M.S. Wolfe, 1999. Host frequency and density effects on powdery mildew and yield in mixtures of barley cultivars. Plant Pathol., 48: 807-816. DOI: 10.1046/j.1365-3059.1999.00398.x

Garrett, K.A. and C.C. Mundt, 2000. Effects of planting density and the composition of wheat cultivar mixtures on stripe rust: an analysis taking into account limits to the replication of controls. Phytopathology, 90: 1313-1321. PMID: 18943371 
Gautam, P. and R. Dill-Macky, 2012. Impact of moisture, host genetics and Fusarium graminearum isolates on Fusarium head blight development and trichothecene accumulation in spring wheat. Mycotoxin Res., 28: 45-58. DOI: 10.1007/s12550011-0115-6

Glover, K.D., J.C. Rudd, R.N. Devkota, R.G. Hall and Y. Jin et al., 2010. Registration of 'Brick' wheat. J. Plant. Regis., 4: 22-27.

Klepper, B., R.W. Rickman, S. Waldman and P. Chevaliar, 1998. The physiological life cycle of wheat: Its use in breeding and crop management. Euphytica, 100: 341-347.

Mass, E.V., S.M. Lesch, L.E. Francois and C.M. Grieve, 1996. Contribution of individual culms to yield of salt-stressed wheat. Crop Sci., 36: 142-149.

Miller, J.D. and J.C. Young, 1985. Deoxynivalenol in an Experimental Fusarium Graminearum Infection of Wheat. Can. J. Plant Pathol., 7: 132-134.

Nerson, H., 1980. Effects of population density and number of ears on wheat yield and its components. Field Crops Res., 3: 225-234.
Pfleeger, T.G. and C.C. Mundt, 1998. Wheat leaf rust severity as affected by plant density and species proportion in simple communities of wheat and wild oats. Phytopathology, 88: 708-714. PMID: 18944944

Pumphrey, M.O., R. Bernardo and J.A. Anderson, 2007. Validating the Fhb1 QTL for fusarium head blight resistance in near-isogenic wheat lines developed from breeding populations. Crop Sci., 47: 200-206. DOI: $10.2135 /$ cropsci2006.03.0206

Scott, P.M., K. Nelson, S.R. Kanhere, K.F. Karpinski and S. Hayward et al., 1984. Decline in Deoxynivalenol (Vomitoxin) concentrations in 1983 ontario winter wheat before harvest. Appl. Environ. Microbiol., 48: 884-886.

Strange, R. N. and Smith, H. 1971. A fungal growth stimulant in anthers which predisposes wheat to attack by fusarium graminearum. Physiol. Plant Pathol. 1: 141-150. DOI: 10.1016/00484059(71)90023-3 